\title{
Risco crescente de melanoma de pele no Brasil.
}

\author{
Increasing risk of cutaneous melanoma in Brazil
}

\author{
Guinar Azevedo e Silva Mendonça*
}

\begin{abstract}
MENDONÇA, G.A. e S. Risco crescente de melanoma de pele no Brasil. Rev. Saúde públ., S. Paulo, 26: 290-4, 1992. A ocorrência de melonoma maligno de pele no Brasil é analisada a partir dos dados de mortalidade disponiveis no Ministério da Saúde e dos dados de incidencia dos seis Registros de Câncer de Base Populacional, localizados em seis capitais brasileiras. Os coeficientes de incidência nessas capitais situam-se em padrões intermediários se comparadas às cifras mundiais. Para o Município de Porto Alegre, uma das capitais estudas, que apresentou os maiores coeficientes de incidência, é feita comparação entre os dados relativos ao periodo 1979-1982 e 1987, constatando-se que houve aumento relativo de $38 \%$ entre homens e de $11 \%$ entre mulheres. Concluiu-se pela necessidade de se conduzir estudos no Brasil entre comunidades de indivíduos de pele clara, os quais apresentam risco potencializado para o desenvolvimento de melanoma, para que sejam definidas medidas especificas e eficazes de controle.
\end{abstract}

Descritores: Melanoma, epidemiologia. Neoplasias cutâneas, epidemiologia. Fatores de risco.

\section{Introdução}

A incidência de melanoma maligno de pele tem aumentado em vários países nos últimos quarenta anos ${ }^{3,6,12,16,20}$. Tal fato vem sendo alvo de preocupação para as autoridades de saúde pública desses países e muitas campanhas de prevenção e diagnóstico precoce têm sido realizadas nos últimos anos. O melanoma cutâneo é potencialmente hoje um câncer que pode ser curado se tratado precocemente $^{16}$, mas sua letalidade ainda é alta nos casos em que a doença é diagnosticada em fase não inicial.

A evidência de que as pessoas de pele clara têm maior pré-disposiçâo para o desenvolvimento deste tipo de câncer é fortalecida ao se verificar que os coeficientes de ocorrência, em populaçōes de raça negra e amarela, são inferiores às de raça branca ${ }^{19}$.

A relação entre o melanoma e a exposição solar ainda não foi totalmente esclarecida ${ }^{7}$, não parecendo ser tão direta como no caso dos outros tumores de pele não-melanocíticos. A hipótese de que o melanoma esteja relacionado a exposições solares intermitentes intensas já foi descrita por muitos autores ${ }^{5,15}$. Outros fatores devem estar envolvidos dentro da etiologia do melanoma cutâneo.

O trabalho rural como os hábitos de lazer

\footnotetext{
* Serviço de Epidemiologia. Coordenação de Programas de Controle de Câncer. Instituto Nacional do Câncer Rio de Janeiro, RJ - Brasil

Separatas/Reprints: G.A. e S. Mendonça - Praça Vermetha, 23 - Sala 320 - 20230-130 - Rio de Janeiro, RJ - Brasil.
}

e de atividades esportivas ao ar livre favorecem enormemente a exposição solar no Brasil. Por outro lado, a grande miscigenação de etnias no país dilui, em parte, o risco de ter pele clara, originária da raça branca ${ }^{17}$.

No entanto, há no país populações de maior risco para o câncer de pele, em geral, representadas por descendentes de europeus. Nas regiōes sudeste e sul, onde foi mais intensa a concentração de imigrantes da Europa Central, existem comunidades que, por razões geográficas, sociais e culturais sofreram pouca ou quase nenhuma miscigenação racial. Conseqüentemente, estas comunidades expressam risco importante, por suas características raciais, para o desenvolvimento de câncer de pele.

A inexistência de informações mais abrangentes e detalhadas sobre as neoplasias malignas de pele no Brasil, impossibilita que se trace perfil real da magnitude do problema.

Assim, pois, o objetivo do presente trabalho é analisar, através dos dados dos Registros de Câncer de Base Populacional, a incidência de melanoma de pele e discutir os fatores de risco mais importantes no Brasil.

\section{A ocorrência no Brasil}

Em 1985, o subsistema de Mortalidade do Ministério da Saúde registrou 462 casos de morte que tiveram, como causa básica, melanoma maligno de pele ${ }^{13}$, e foram classificados segundo o código 173 da Classificação Inter- 
nacional de Doenças (CID), 9 revisão ${ }^{14}$. Este número representou $0,66 \%$ do total de mortes por câncer, ocupando o vigésimo sexto posto entre as demais neoplasias malignas. Verificou-se, ainda, que, entre 1977 e 1985 , os coeficientes de mortalidade por melanoma de pele não apresentaram grandes variações indicando um risco de morte para todas as idades em torno de 0,40 por 100.000 habitantes ${ }^{13}$ (Tabela 1). Cabe ressaltar que a subnotificação da informação sobre mortes que tiveram como diagnóstico melanoma deve ser importante, provavelmente tendo em conta a evolução rápida desse tipo de neoplasia e sua alta letalidade. É possivel, assim, que parte dos óbitos por melanoma maligno de pele esteja incluída entre o grupo classificado como causas de origem mal definidas ou mesmo entre os tumores malignos de origem primária desconhecida.

A tabela 2 mostra os coeficientes de incidência de melanoma de pele, segundo a Clas-

Tabela 1 - Número de óbitos segundo sexo e ceficiente de mortalidade por melanoma maligno de pele (CID=172) no Brasil, 1977/1985

\begin{tabular}{lcccc}
\hline $\begin{array}{l}\text { Número de obitos } \\
\text { Ano }\end{array}$ & \multicolumn{4}{c}{$\begin{array}{c}\text { Taxa de mortalidade* } \\
\text { (por 100.000 hab.) } \\
\text { Total }\end{array}$} \\
\hline 1977 & 181 & 135 & 316 & 0,38 \\
1978 & 204 & 152 & 356 & 0,40 \\
1979 & 251 & 152 & 403 & 0,41 \\
1980 & 239 & 160 & 399 & 0,39 \\
1981 & 279 & 185 & 464 & 0,43 \\
1982 & 247 & 193 & 440 & 0,39 \\
1983 & 256 & 193 & 449 & 0,38 \\
1984 & 288 & 176 & 464 & 0,38 \\
1985 & 269 & 193 & 462 & 0,40 \\
\hline
\end{tabular}

- Coeficiente calculado com base na população estimada dos municípios com informação regular Fontes: Estatísticas de Mortalidade - Brasil, 1977.1985

sificação Internacional de Doenças (CID - 9a revisão), dos Registros de Câncer de Base Populacional de quatro capitais brasileiras comparadas às regiões do mundo de mais altas e mais baixos indices, em torno de $1980^{19}$.

Dentro do quadro mundial tais cifras se situam dentro de padrão intermediário. As taxas de São Paulo e Porto Alegre são superiores às encontradas em Recife e Fortaleza. Explicação para isto seria o fato de ter sido justamente na região sul e sudeste do Brasil, onde se concentraram majoritariamente os imigrantes europeus o que deu origem a populações com grandes
Tabela 2 . Coeficiente de incidência de melanoma ma. ligno de pele $(C I D=172)$ em quatro capitais brasileiras $e$ em outras regioes do mundo, 1978/1982.

\begin{tabular}{lcc}
\hline \multirow{2}{*}{ Regiōes } & \multicolumn{2}{c}{$\begin{array}{c}\text { Taxa de incidência(a) } \\
\text { (por 100.000 hab.) }\end{array}$} \\
\cline { 2 - 3 } & Homens & Mulheres \\
\hline Queensiand, Austrália (b) & 30,9 & 28,5 \\
Hawaí: população branca (b) & 22,7 & 18,8 \\
Nova Zelândia: populaçāo & 15,6 & 21,4 \\
branca (b) & & \\
Los Angeles, EUA: & 12,4 & 10,9 \\
população branca (b) & & \\
Atlanta, EUA: população & 12,3 & 10,3 \\
branca (b) & & \\
& & \\
Porto Alegre (1979-82) & 3,7 & 2,7 \\
São Paulo (1978) & 3,5 & 4,0 \\
Recife (1980) & 1,6 & 2,1 \\
Fortaleza (1978-82) & 1,3 & 1,0 \\
& & \\
Shangai, China (c) & 0,3 & 0,1 \\
Nagasaki, Japão (c) & 0,2 & 0,2 \\
Bombay, Índia (c) & 0,2 & 0,2 \\
Madras, Índia (c) & 0,1 & - \\
\hline
\end{tabular}

(a) Coeficientes padronizados segundo população mundial

(b) Regiōes de mais altas taxas

(c) Regiōes de mais baixas taxas

Fontes: Waterhouse, J. e $\mathrm{Col}^{19}$ (1987)

contingentes de individuos de cor branca.

A Tabela 3 apresenta dados referentes aos registros de Fortaleza para o ano de 1983 e Porto Alegre para 1987 sobre a incidência de melanoma de pele. Encontram-se também na mesma Tabela dados de dois outros Registros de Câncer de Base Populacional criados mais recentemente em Goiânia e Belém ${ }^{10}$, que dispõem de dados relativos aos anos de 1987 e 1989, respectivamente.

Comparando-se o coeficiente de incidência médio anual do período 1979-1982 com o

Tabela 3 - Coeficientes de incidência de melanoma de pele $(C \mid D=172)$ em Belém, Fortaleza, Porto Alegre $\theta$ Goiânia, 1983/1988.

\begin{tabular}{lcc}
\hline \multirow{2}{*}{ Municípios } & \multicolumn{2}{c}{$\begin{array}{c}\text { Taxas de incidència por } \\
100.000 \text { hab }^{*}\end{array}$} \\
\cline { 2 - 3 } & Homens & Mulheres \\
\hline Belém (1989) & 1,4 & 0,6 \\
Fortaleza (1983) & 1,7 & 1,1 \\
Porto Alegre (1987) & 5,1 & 3,0 \\
Goiânia (1987) & 0,8 & 2,6 \\
\hline
\end{tabular}

* Coeficientes padronizados segundo população mun. dial.

Fonte: Instituto Nacional do Câncer ${ }^{10}$ (1991) 
coeficiente de 1987 de melanoma de pele em Porto Alegre, Município que mostrou as maiores cifras dentre as demais apresentadas, notase um incremento relativo de $38 \%$ no sexo masculino e de $11 \%$ no sexo feminino. Esta comparação não pode ser considerada ideal por ser feita dentro de breve período, sendo que o primeiro referencial tem como base dados de quatro anos e o segundo refere-se a dados de apenas um ano. $\mathrm{Na}$ impossibilidade de obtenção de outras informações sobre a incidência da doença, esta comparação pode trazer alguma indicação de tendência.

\section{Fatores de risco}

O papel da radiação solar no aparecimento dos carcinomas basocelulares e espinocelulares já está estabelecido tanto epidemiológica quanto experimentalmente ${ }^{8}$. No caso de melanoma cutâneo esta relacão ainda não é totalmente conhecida ${ }^{20}$.

Existem, porém, muitas evidências de que o aumento de incidência de melanoma nos últimos 50 anos teve relação com o aumento de exposição a raios solares ${ }^{2}$.

Entre os pacientes com melanoma de pele, a distribuição anatômica das lesões não se dá, prioritariamente, em áreas expostas ao sol como se observa entre os portadores de epiteliomas basocelulares e tumores espinocelulares. Observa-se, porém, que o risco de melanoma, em áreas da pele que são pouco expostas ao sol, aumenta em decorrência de exposiçōes intermitentes e intensas ao sol ${ }^{9}$. Alguns estudos, por exemplo, mostraram que trabalhadores que não se expõem ao sol tiveram riscos iguais ou maiores do que os trabalhadores com atividades externas 1 .

$\mathrm{Na}$ Europa, por exemplo, os coeficientes de incidência são mais altos no norte, justamente em regiões mais distantes do Equador, onde existem mais indivíduos de pele clara ${ }^{18}$.

Vários autores vêm se dedicando à condução de pesquisas com o objetivo de elucidar a etiologia do melanoma maligno de pele. Rhodes e col. ${ }^{16}$ afirmam que os fatores de risco mais importantes na etiologia do melanoma, em ordem decrescente, seriam:

$\left.1^{\circ}\right)$ presença de nevo transformado ou em transformação; $2^{\circ}$ ) idade adulta; $3^{\circ}$ ) existência de lesões pigmentares irregulares (inclusive nevo displásico e lentigo maligno); $4^{\circ}$ ) existência de um nevo congênito; $5^{\circ}$ ) ser de raça caucasiana; $6^{\circ}$ ) história de melanoma cutâneo prévio; $7^{\circ}$ ) relato de melanoma cutâneo na fa- milia; $8^{\circ}$ ) imunodepressão $\left.9^{\circ}\right)$ sensibilidade ao sol e $10^{\circ}$ ) exposição solar excessiva.

Estudo de caso-controle que analisou todos os pacientes que tiveram diagnóstico de melanoma na Escócia, em 1987, identificou como principais fatores de risco: grande quantidade de nevos pigmentados menores que $2 \mathrm{~mm}$; tendência a genodermatose; presença de nevos de até $5 \mathrm{~mm}$ de diâmetro com bordo irregular ou com pigmentação irregular ou inflamação e história de queimadura solar intensa em qualquer época da vida ${ }^{12}$. Na Austrália, Green e col. ${ }^{8}$, a partir também de estudo de caso-controle, concluíram que havia uma relação dose-resposta significante entre queimaduras solares e melanoma cutâneo sendo o risco relativo de 1.5 para dois a cinco episódios e de 2.4 para seis ou mais. Elwood e col. ${ }^{5}$ analisando 595 pacientes com melanoma comparados a controles populacionais, mostraram que as curacterísticas individuais de pigmentação e a reação da pele ao sol estão mais associados ao risco de ter melanoma do que os relatos prévios de queimaduras solares e bronzeamentos.

Estudo italiano mostrou que trabalhar em ambientes externos e ter história prévia de queimaduras solares estão positivamente relacionados com o risco de desenvolver melanoma em áreas anatômicas do corpo expostas ao sol e negativamente em áreas protegidas ${ }^{3}$.

Green e $\mathrm{O}^{\prime}$ Rourke $^{7}$ verificaram que havia uma relação direta entre a existência de lesões actínicas e melanoma, o que mostra que este tipo de tumor ocorre em indivíduos com pele suscetivel ao dano actínico.

Alguns outros estudos avançaram no sentido de caracterizar que indivíduos que se expõem continuamente ao sol não apresentam risco de desenvolver melanoma ${ }^{15}$ ou apresentam até uma redução de risco ${ }^{4,6}$.

Os achados de uma pesquisa realizada com enfermeiras americanas, entre 1921 e 1946, mostraram que as mulheres com pele resistente ao sol não apresentaram tisco de desenvolver melanoma cutâneo ${ }^{20}$.

Kirkpatrick e col. ${ }^{11}$ analisando os dados de onze Registros de Câncer de base populacional nos Estados Unidos chegaram a conclusão de que na terceira idade os melanomas não-Hutchinson, que aparecem em regiões do corpo protegidas do sol, foram 4,5 vezes mais comuns nos grupos sociais mais baixos economicamente. Isto sugere, segundo os autores, que a exposição crônica e prolongada ao sol tem implicação no aparecimento deste tipo de melanoma em pessoas idosas. 


\section{Conclusões}

Considerando que tanto as caracteristicas individuais para o tipo de pele como a exposição aos raios utravioleta têm implicações na etiologia do melanoma, a caracterização e a forma de interação destes dois fatores de risco no Brasil merecem uma análise cuidadosa.

Não há dúvida de que os indivíduos de origem européia que vivem em clima tropical ou temperado apresentam um risco elevado de desenvolver câncer cutâneo. Grande parte dos imigrantes europeus que se estableleceram no Brasil e seus descendentes se dedicaram ao trabalho rural. É possível encontrar-se nas regiōes sudeste e sul comunidades agrícolas com grande concentração de pessoas de pele clara. Esses indivíduos expõcm-se ao sol continuadamente, muitas vezes desde a primeira infância. Além disto, a exposição solar decorrente de atividades esportivas de lazer é bastante comum em todos os extratos sociais de todo o território nacional. Sendo assim, a potencialização de riscos para câncer de pele está presente na realidade brasileira o que justifica a tomada de medidas eficazes que possam prevenir e incentivar o diagnóstico e tratamento precoce dos tumores de pele.

Apesar de todos os esforços na busca de novas terapêuticas para melanoma de pele, a letalidade ainda é altíssima quando a deteç̧ão é feita tardiamente. Existem relatos de que campanhas de rastreamento tiveram como resultado a capitação de um maior número de casos com lesōes em estágio inicial na Austrália e Escócia ${ }^{12}$.

Comprovadamente, a espessura da lesão é o mais importante fator prognóstico para o melanoma.

Como o melanoma é um tipo de câncer pouco frequiente se comparado a outros tipos como o de pulmão ou de mama, as medidas preventivas terão mais êxito se forem dirigidas diretamente aos grupos de maior risco. Torna-se, assim, necessário a condução de estudos no Brasil que possam idientificar estes grupos de alto risco e a partir daí estabelecerse política de controle que contemple medidas adequadas e especificas.

MENDONÇA, G.A. e S. [Increasing risk of cutaneous melanoma in Brazill. Rev. Saúde públ., S.Paulo, 26: 290-4, 1992. The occurrence of cutaneous malignant melanoma in Brazil is analysed on the basis of available mortality data from the National Ministery of Health and from the incidence data of six Population-based Cancer Registries (Recife, Fortaleza, S.Paulo, Porto Alegre,
Goiânia e Belém). The incidence in these State capitals has an intermediate pattern if compared to the world pictures. For Porto Alegre, the capital that had the highest rates, a comparison between the periods 1979-1982 and 1987 showed a proportional increases of $38 \%$ among males and $11 \%$ among females. The conclusion is reached that it is necessary to undertake studies in Brazil among fairskinned people from particular communities which may show a potentialized risk for the development of cutaneous melanoma in order to be able to define what kind of specific control actions should be developed.

Keywords: Melanoma, epidemiology. Skin neoplasms, epidemiology. Risk factors.

\section{Referências Bibliográficas}

1. COOKE, K.R et al. Socio-economic status, indoor and outdoor work, and malignant melanoma. Int.J. Cancer, 34: 57-62, 1984.

2. COUNCIL ON SCIENTIFIC AFFAIRS. Harmful effects of ultraviolet radioation. J.Amer.med.Ass., 262: 380-4, 1989.

3. CRISTOFOLINI, M. et al. Risk factors for cutaneous malignant melanoma in a northem Italian population. Int. J. Cancer. 39: 150-4, 1987.

4. ELWOOD, J.M. et al. Cutaneous melanoma in relation to intermittent and constant sun exposure: the Westem Canada Melanoma Study. Int.J.Cancer, 35: 427-33, 1985.

5. ELWOOD, J.M. et al. Sunbum, suntan and the risk of cutaneous malignant melanoma: the Westem Canada Melanoma Study. Brit. J. Cancer, 51: 543-9, 1985.

6. GALLAGHER, R.P. et al. Is chronic sunlight exposure important in accounting for increases in melanoma incidence? Int. J. Cancer, 44: 813-5, 1989.

7. GREEN, A. \& O'ROURKE, M.G.E. Cutaneous malignant melanoma in association with other skin cancers. J.nat. Cancer Inst., 74: 977-80, 1985.

8. GREEN, A. et al. Sunbum and malignant melanoma. Brit. J. Cancer, 51: 393-7, 1985.

9. HOLMAN, C.D.J. et al. A theory of the etiology and pathogenesis of human cutaneous malignant melanoma. J. nat. Cancer Inst., 71: 651-6, 1983.

10. INSTITUTO NACIONAL DE CÂNCER/PRO-ONCO. Câncer no Brasil: dados dos registros de base populacional. Rio de Janeiro, 1991.

11. KIRKPATRICK, C.S. et al. Melanoma risk by age and socio-economic status. Int.J.Cancer, 46: 1-4, 1990.

12. MACKIE, R.M. et al. Personal risk-factor chart for cutaneous melanoma. Lancet, 2: 487.93, 1989.

13. MINISTERIO DA SAÚDE Estatística de mortalidade: Brasil; 1977-1985. Brasília, 1982-1988.

14. ORGANIZAÇÃO MUNDLAL DA SAÚDE. Mamual de classificação estatística internacional de doenças, lesōes e causas de óbitos: 94. revisão; 1975. São Paulo, Centro da OMS para Classificação de Doenças em Português, 1979. 2v.

15. OSTERLIND, A. et al. The Danish case-control study of cutaneous malignant melanoma. II - Importance of UV light exposure. Int.J.Cancer, 42: 319-24, 1988.

16. RHODES, A.R. et al. Risk factors for cutaneous melanoma: a pratical method of recognizing individuals. J.Amer.med.Ass, 285: 3146-54, 1987.

17. SCHOTTENFELD, D. \& FRAUMENI, J.F., eds Cancer epidemiology and prevention. Philadelphia, Saunders, 1982. 
18. TOMATIS, L. et al. Cancer: causes, occurrence and control. Lyon, IARC, 1990. (IARC Scientific Publication, 100).

19. WATERHOUSE, J. et al. Cancer incidence in five continents. Lyon, IARC, 1987. v. 5. (IARC Scientific Publication, 88).
20. WEINSTOCK, M.A. et al. Melanoma and the sun: the effect of swimsuits and a "healthy" tan on the risk of nonfamilial malignant melanoma in women. Amer.J.Epidem., 134: 462-70, 1991.

Recebido para publicação em 3/2/1992

Reapresentado em 21/5/1992 Aprovado para publicação em 25/5/1992 